\title{
DYNAMICAL ASPECTS OF THE TAURID METEOR COMPLEX
}

\author{
J. ŠTOHL and V. PORUBČAN \\ Astronomical Institute of the Slovak Academy of Sciences, Interplanetary Matter \\ Division, \\ 842 28 Bratislava, Czech and Slovak Federal Republik
}

October 11, 1991

\begin{abstract}
Unusually long activity of the Taurid meteor complex, extending over 3-5 months according to new estimations based on various orbital similarity criteria, has been evoking controversies about the possible origin and dynamical evolution of this unique complex of meteor streams. It even casts doubts on the reality of the extension of the complex. In the present paper orbital elements and the extension of the Taurid meteor complex are re-examined on the bases of the most precise photographic meteor orbits available from the IAU Meteor Data Center in Lund. The results are evaluated and discussed from the viewpoint of various proposals on the origin and dynamical evolution of the complex.
\end{abstract}

Key words: meteor streams, Taurid meteor complex, D-criterion

\section{Introduction}

The Taurid meteor complex (hereafter TMC) consists of several mutually associated meteor streams, regarded once as completely independent. Radiants of meteors belonging to these streams form a continuous long chain along the ecliptic, with its central and most active part falling on the Taurus constellation and producing thus well known Taurid shower. According to early observations this central part, i.e. the Taurids are active mostly for about one month, from October 26 to November 22 (Lovell 1954).

Activity of the Taurid shower has a very complex character, as was recognized by Denning from visual observations as early as in late twenties (cf. Lovell (1954), p.298). He was able to identify 13 active centers of meteors in the Taurus and Aries constellations. First photographic observations of meteors by Whipple (1940) confirmed multiplicity of the Taurids radiants. This fact together with the long endurance of the Taurid shower led Whipple to his remark that "the dilution of the Taurid stream has disguised its character as one of the more important known showers", and that "the total population of the associated streams is probably comparable with that of any of the generally recognized streams". A detailed analysis of the Harvard photographic orbits of meteors carried out by Wright and Whipple (1950) not only confirmed the complex character of the Taurid activity, but also revealed that the stream consists of two branches, i.e. of the Northern and the Southern Taurids. The analysis has also shown that the more concentrated Southern Taurids may form a continuous stream with the Southern branch of the Arietid shower, which would mean that they both are combined into one moving radiant, as was proposed by Wright and Whipple in their paper.

Whipple's suggestion about the association of the Taurids with other meteor streams has been perfectly confirmed on much larger scale than the one he had at 
his disposal. Several papers were devoted to the questions which meteor streams are associated with the Taurid stream and to what extent these associations forming TMC can be taken as real: Sekanina (1973, 1976), Drummond (1981), Štohl (1986), Olsson-Steel (1987), Porubčan and Štohl (1987a), (1987b), Babadzhanov and Obrubov (1989), Babadzhanov et al.(1990), Štohl and Porubčan (1990), Steel et al. (1991).

It is exactly the long duration of the TMC activity, caused by large spread of its orbits, which makes the origin and dynamical evolution of TMC an intriquing problem. If the extremely long duration of TMC is confirmed, then it would become very difficult if not impossible to accept just one comet, namely P/Encke as the only progenitor of the whole TMC. In fact, several comets and asteroids have been proposed as being closely associated with the TMC: Whipple and Hamid (1952), Wasson and Wetherill (1979), Galibina and Kastel (1982), Napier (1983), Clube and Napier (1986), Olsson-Steel (1987), Porubčan and Štohl (1987b), Clube and Asher (1990), Steel et al. (1991). Solution of this problem seems to be of a fundamental meaning for the origin and dynamical evolution of the minor bodies in the Solar System in general. It is the aim of the present paper to scrutinize the reality and extension of the TMC.

\section{Orbital associations within TMC}

To obtain as reliable conclusion as possible about meteors belonging to the TMC we have included into our analysis all precise photographic orbits of meteors available to the end of 1990 from the IAU Meteor Data Center in Lund (Lindblad (1987)). In comparison with our previous analysis (Štohl and Porubčan (1990)) the number of orbits is substantially enlarged, from the earlier number of 2760 to the present total of 3518 orbits.

Radar orbits, though of much larger number, have not been searched because of their relatively low accuracy, in spite of the fact that they are observable during both the preperihelion and postperihelion encounter of TMC with the Earth's orbit. By taking into account photographic orbits we are restricted to the investigation of the preperihelion encounter observable during night hours only. We are convinced that this restriction is compensated by higher precision of the photographic orbits and more reliable outcome of the analysis.

Method for finding out the associations within the TMC used in the present analysis is identical with that used in our previous papers (Porubčan and Stohl (1987a), (1987b), Štohl and Porubčan (1990)). As the first approach mean orbits were calculated of all meteors which were indicated as the Northern or Southern Taurids in the original data on meteor orbits (Porubčan (1978)). To these mean orbits of the two branches of the Taurids a strong Southworth-Hawkins criterion $\mathrm{D}=0.18$ was applied on all meteor orbits within the period from September 1 to January 31. We have excluded all orbits with $Q>5.1 \mathrm{AU}$ and with an element 
deviated to more than 3 std. dev. from the particular mean orbit. As a result we have identified 49 orbits of the Northern and 95 orbits of the Southern Taurids. These meteors we accept as the core of the whole Taurid meteor complex.

As the second approximation for finding out the most reliable associations of meteor orbits and streams with the TMC, variations of the orbital elements of the Taurids had to taken into account. As was demonstrated in our previous paper (Štohl and Porubčan (1987)), in the case of Taurids calculations of the orbital associations using the D-criterion applied only to the mean orbits of the Taurid branches would give ambiguous results. This effect follows from the very large spread of their orbits and from systematic changes of their orbital elements in the course of the extraordinary long duration the Taurid activity. We thus assume that the mean orbits of the Northern and Southern Taurids undergo systematic change during their activity, in accordance with the variations of their orbital elements. For calculation of such "varied mean orbits" corresponding to the longitude of the ascending node of a particular meteor orbit, we have accepted the values of variations derived in our earlier paper for the core of TMC as defined above (cf. Štohl and Porubčan (1990)).

For this second approximation we could chose a larger value of the D-criterion, namely $D=0.25$. This value is fully substantiated taking into account very large dispersion of the orbital elements of the Taurids. In comparison with the previous search our present investigation is restricted more realistically to the period from September 1 to January 31. Within this period the D-values were calculated for each meteor orbit and the corresponding "varied mean orbit". For the limit $D=0.25$ we have identified 211 meteors which can be accepted as members of the TMC, from which 74 and 137 meteors belong to the Northern and Southern branches of the TMC, respectively. Their activities extend from September 8 to January 23 for members of the Southern branch and from September 1 to January 16 for the ones of the Northern branch of TMC.

TABLE I

Mean orbits of the Taurid meteor complex

\begin{tabular}{|c|l|c|c|c|c|c|c|c|}
\hline Branch & & $\mathrm{a}$ & $\mathrm{q}$ & $\mathrm{e}$ & $\mathrm{i}$ & $\omega$ & $\Omega$ & $\mathrm{n}$ \\
\hline Northern & mean & 2.19 & 0.356 & 0.828 & 3.6 & 294.8 & 225.8 & 74 \\
& std.dev. & 0.56 & 0.080 & 0.058 & 1.9 & 9.6 & 34.7 & \\
& & & & & & & & \\
Southern & mean & 2.18 & 0.383 & 0.812 & 5.2 & 112.1 & 41.6 & 137 \\
& std.dev. & 0.82 & 0.104 & 0.063 & 1.6 & 12.9 & 29.7 & \\
\hline
\end{tabular}

In Table I we present mean orbits of the Northern and Southern branches of the TMC, calculated for all meteors identified in the second approximation as members of the TMC. The values given in Table I we propose as the best estimates of the mean orbits of the whole TMC. 
Radiants of the members of TMC are shown in upper part of Fig.1. The Northern and Southern branches of the TMC are evident throughout whole period of their activity. It is seen that the radiants are dispersed along the ecliptic within about $130^{\circ}$ of the right ascension (R), from $354^{\circ}$ to $122^{\circ}$ for the Northern branch and from $3^{\circ}$ to $118^{\circ}$ for the Southern branch. The core of the TMC, i.e. the classical Taurids with $\mathrm{D}<0.18$ have their radiants dispersed in $\mathrm{R}$ from September 25 to November 23 for the Southern Taurids and from October 9 to November 27 for the Northern Taurids.
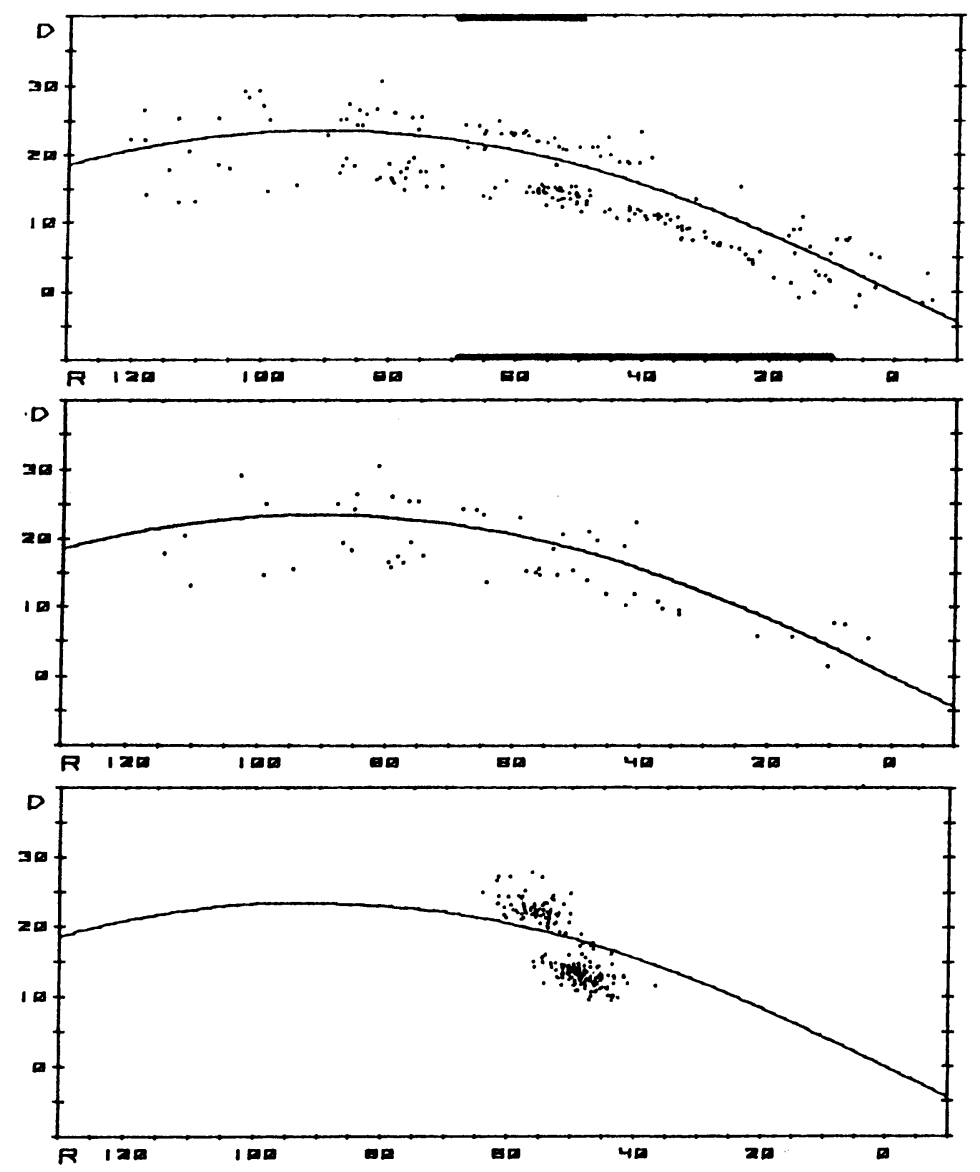

Fig. 1. Radiants of the Taurid complex meteors

Affiliation with TMC of all the 211 meteors is also supported by the lower parts of Fig.1. It is known that the Taurids are very rich on bright fireballs. Middle part of 
Fig. 1 shows radiants of TMC fireballs brigther than $-3^{m}$. It confirms that the TMC fireballs activity continues practically throughout the whole TMC period. Orbits of the TMC fireballs are shown in projection onto ecliptical plane in Fig.2. Their dispersion is to be compared with the dispersion of all Taurid orbits as presented e.g. in Fig. 1 of Porubčan and Sttohl (1987b). We note that the dispersion of orbits in both cases is consistent. In the lower part of Fig.1 all 211 TMC radiants are shown with their positions reduced to the mean longitude of the ascending or descending nodes of the Northern and Southern branches of TMC, respectively. It is evident that all the radiants fall into two areas with dimensions approximately $15^{\circ} \times 10^{\circ}$, confirming thus inner compatibility of the corresponding orbits with the TMC.

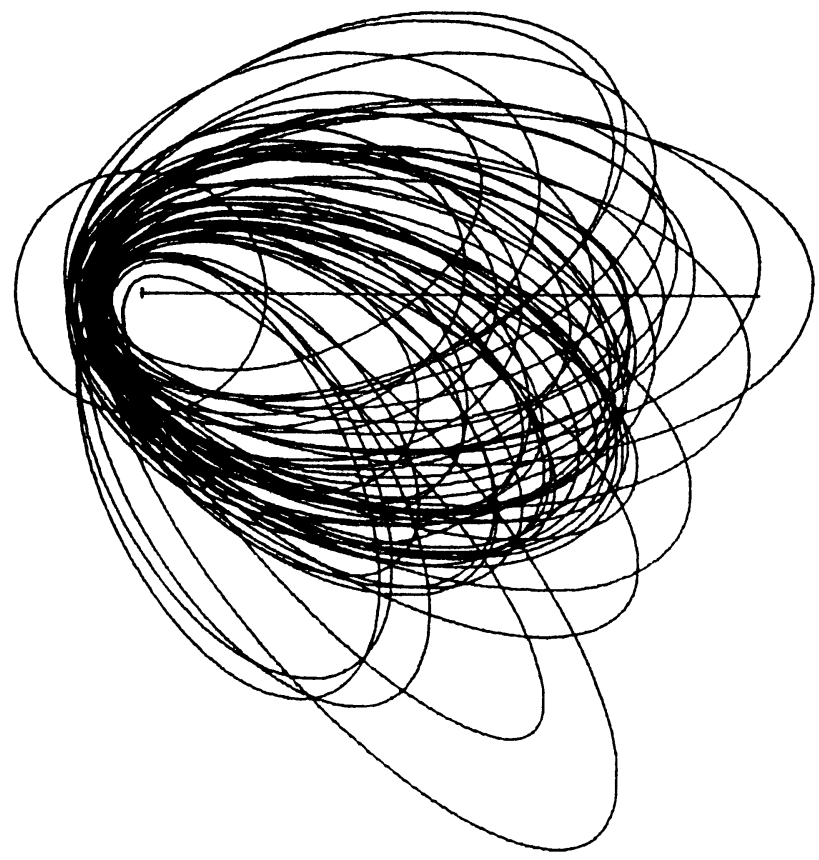

Fig. 2. Orbits of the TMC fireballs projected onto the ecliptic plane

\section{Meteor streams of the TMC}

Many of meteors identified above as members of the TMC had been denoted originally as members of various showers: Arietids and Piscids at the beginning of TMC activity, and $\chi$ Orionids (identical with Lindblad's (1971) $\chi$ Geminids) and $\rho$ Geminids (identical with Sekanina's (1973) and (1976) Canids) at the end of TMC activity. High probability of these streams associations with TMC is supported by 
the corresponding very low D-values as derived by Štohl and Porubčan (1990). Their D-values are presented in Table II together with the elements of the observed mean orbits of the particular streams $(\mathrm{O})$ and corresponding calculated "varied mean orbits" of TMC (C). For the mean obseved orbits of the streams Kronk's (1988) compiled values were accepted, with the exception of the mean orbit of the $\rho$ Geminids which was taken as an average from the orbits by Lindblad (1971), and Sekanina (1973) and (1976). By N and S in Table II the Northern and Southern branches are denoted, respectively.

TABLE II

Minor showers belonging to the Taurid complex

\begin{tabular}{|l|c|c|c|c|c|c|c|}
\hline Shower & $\mathrm{D}$ & & $\mathrm{q}$ & $\mathrm{e}$ & $\mathrm{i}$ & $\omega$ & $\pi$ \\
\hline N Piscids & 0.058 & $\mathrm{O}$ & 0.271 & 0.853 & 6.5 & 306.6 & 121.3 \\
N Taurids & & $\mathrm{C}$ & 0.280 & 0.856 & 3.9 & 304.3 & 119.0 \\
& & & & & & & \\
S Arietids & 0.054 & $\mathrm{O}$ & 0.280 & 0.846 & 6.4 & 125.2 & 140.6 \\
S Taurids & & $\mathrm{C}$ & 0.304 & 0.833 & 5.7 & 122.1 & 137.5 \\
& & & & & & & \\
N $\chi$ Orionids & 0.185 & $\mathrm{O}$ & 0.528 & 0.739 & 2.4 & 275.5 & 176.5 \\
N Taurids & & $\mathrm{C}$ & 0.426 & 0.807 & 2.6 & 285.8 & 186.8 \\
& & & & & & & \\
S $\chi$ Orionids & 0.052 & $\mathrm{O}$ & 0.460 & 0.798 & 6.4 & 101.5 & 179.4 \\
S Taurids & & $\mathrm{C}$ & 0.484 & 0.782 & 5.1 & 98.8 & 176.7 \\
& & & & & & & \\
$\rho$ Geminids & 0.085 & $\mathrm{O}$ & 0.647 & 0.709 & 4.5 & 260.4 & 196.6 \\
S Taurids & & $\mathrm{C}$ & 0.594 & 0.750 & 4.8 & 264.5 & 200.7 \\
\hline
\end{tabular}

The agreement between the observed and calculated orbits in all cases (except of the Northern $\chi$ Orionids) is excellent. It means that these streams should be taken as regular parts of TMC in the sense proposed by Wright and Whipple (1950). All these streams are thus combined into one moving radiant consisting of two branches.

Support for this conclusion also follows from the investigation of individual meteor associations with each of the TMC streams listed in Table II. Periods of the streams activity with corresponding numbers of meteors associated with them within the D-values 0.25 and 0.20 , respectively, are presented in Table III. In the case of the $\rho$ Geminids the same mean orbit from Table II was applied to both the Northern and Southern branches. Particular meteors found to be associated with the $\rho$ Geminids were attributed to the Northern or Southern branch according to their ascending or descending nodes.

As could be expected from the previous conclusion, the streams are overlapping each other successively at the ends of their activities. In the case of the more abundant Southern branch the overlapping is especially evident: 59 meteors out of the 66 
Southern Arietids, e.g., at the same time are members of the Southern Taurids, out of their total number of 86 . The sequence of the TMC streams from Piscids/Arietids through Taurids and $\chi$ Orionids to $\rho$ Geminids within both branches appears to be thus confirmed.

TABLE III

Activity of the showers within TMC

\begin{tabular}{|l|c|c|c|}
\hline Shower & Period & $\mathrm{N}(D<0.25)$ & $\mathrm{N}(D<0.20)$ \\
\hline N Piscids & Sep 1 - Oct 12 & 14 & 13 \\
N Taurids & Oct 9 - Nov 27 & 30 & 21 \\
N $\chi$ Orionids & Nov 17 - Jan 15 & 20 & 16 \\
N $\rho$ Geminids & Dec 27 - Jan 29 & 10 & 7 \\
\hline S Arietids & Sep 15- Nov 11 & 66 & 33 \\
S Taurids & Sep 25 - Nov 23 & 86 & 74 \\
S $\chi$ Orionids & Nov 20 - Dec 28 & 41 & 28 \\
S $\rho$ Geminids & Dec 28 - Jan 23 & 6 & 3 \\
\hline
\end{tabular}

\section{Problems of the TMC dynamics and origin}

Extremely long duration of the activity of the TMC, large dispersion of its orbits and complexity of its structure does not allow any simple model of its origin and dynamical evolution. Whipple has pointed out to the association of the Taurids with $\mathrm{P} /$ Encke comet, and by studying dynamical evolution of the orbits of the Taurid meteors with Hamid (Whipple and Hamid (1952)) he had to accept an assumption that a part of the stream originated from P/Encke about 4700 years ago and a part from another hypothetical body (resulting from a catastrophic collision of P/Encke with a main-belt asteroid) about 1400 years ago. Without this catastrophic hypothesis it was impossible to explain spread of the orbits at normal low velocity ejection of meteoroids from the parent body. And still, meteor orbits they had at their disposal at that time had their radiants dispersed within $20^{\circ}$ of the ecliptic longitude only (shown by a heavy line on the upper axis in Fig.1).

As was demostrated in the previous sections, the dispersion along the ecliptic longitude of the Taurids and entire TMC orbits available at present appears to be several times larger, extending over $130^{\circ}$ in the ecliptic longitudes. Problem of the origin and dynamical evolution of the TMC thus becomes even more acute.

Attempts to explain the origin of the TMC from a single parent body do not lead to satisfactory results. Jones (1986) performed extensive calculations of the Taurids orbital evolution originated from $\mathrm{P} /$ Encke, taking into account planetary perturbations, but neglecting initial spread in orbits. To explain the dispersion of the Taurid stream he obtained an age of the stream of the order of $10^{5}$ years. We note that for his analysis Jones had only accepted the Taurid shower duration of 
about 77 days as given by Cook (1973). If the duration of the entire TMC is accepted, the age would become still higher.

Taking into account also the initial spread of the orbits, corresponging to the ejection velocities up to $300 \mathrm{~m} / \mathrm{s}$, with assumption of the origin from P/Encke only, Babadzhanov et al. (1990) derived a shorter age of the TMC, about 4700 years for its core, the Taurids, and 8000 - 18000 years for the Piscids and $\chi$ Orionids. At deriving these values, they only took into account orbits corresponding to the spread of the longitudes of perihelion $\pi$ in between $110^{\circ}-185^{\circ}$. For the entire TMC, however, they are spread within the interval $101^{\circ}<\pi<217^{\circ}$ according to the present investigation. The age of the entire TMC with the assumption of a sole parent body would be therefore higher, well over $10^{4}$ years.

If we accept the age of about $10^{4}$ years as the limit for a stream to remain coherent, a realistic model of the origin and orbital evolution of the entire TMC would demand several co-parent bodies. Steel et al. (1991) have examined most plausible models of the TMC origin. Though they took a more conservative approach and only included into their analysis the core of the TMC (shown by heavy line on the lower axis of upper Fig.1) they still came to the conclusion about multiple parent bodies. In their improved model the TMC has resulted from the decay and desintegration of very large progenitor. The decay occurs not only as a consequense of normal ejection of meteoroids from a single nucleus, but also as a consequence of the splitting of large fragments from the original comet, followed perhaps by catastrophic fragmentation in the asteroid belt. Some of them may still exist as P/Encke and Apollo-type asteroids. Support for such a continuing process of at least some meteor streams formation can be seen in sudden outbursts observed, e.g., during the Leonids and Lyrids activities (Porubčan and Štohl (1991)).

Let us remind that several asteroids have been proposed as associated with the TMC: 2201 Oljato, 2212 Hephaistos (1978 SB), 4197 (1982 TA), 1984 KB, 5025 P-L (Wasson and Wetherill (1979), Galibina and Kastel (1982), Napier (1983), Clube and Napier (1986), Olsson-Steel (1987)). Our detailed analysis of the associations of AAA asteroids with meteors on the basis of precise photographic orbits of individual meteors confirmed very close association only of the following asteroids with the TMC: 2201 Oljato, 5025 P-L, 1984 KB and 1991 BA (Porubčan et al. (1991)). All of them are associated with the Southern branch of TMC, 2201 Oljato with the Southern $\chi$ Orionids (within Dec.13-28), 5025 P-L and 1984 KB with the Southern Taurids (within Nov.5-11 and Nov.9-15, respectively), 1991 BA with the Southern $\rho$ Geminids (within Jan.15-28).

\section{Concluding remarks}

An orbital association of the asteroids with the TMC does not necessarily mean their generic relation. To confirm such a relation an analysis of the dynamical evolution and physical characteristics of all bodies involved is needed. On the other hand, 
extremely long activity of the TMC, extending over four months according to our analysis, seems to be confirmed. A few co-parent bodies are therefore demanded. A model of only a single parent body is not acceptable, since it would demand either very high ejection velocities of meteoroids (up to $3 \mathrm{~km} / \mathrm{s}$ ) or very long age of the TMC (up to $10^{5}$ years). The model proposed by Steel et al. (1991) seems to be fully acceptable. In this case the TMC core could originate directly from P/Encke about $5 \times 10^{3}$ years ago, while the TMC outskirts could have been formed by disintegration of the secondary bodies, presumably the asteroids associated with the TMC, which in this case are devolatilized fragments of the original cometary nucleus.

\section{Acknowledgements}

This work was supported by the Slovak Academy of Sciences Grant No. 493/1991.

\section{References}

Babadzhanov, P.B. and Obrubov, Yu.V.: 1989, 'Dynamics and spacial shape of short-period meteoroid streams', in D. McNally (ed.), Highlights of Astronomy 8, 287-293.

Babadzhanov, P.B., Obrubov, Yu.V. and Makhmudov, N.: 1990, 'Meteor showers of P/Encke', Astron. Vestnik 24, 18-28 (in Russian).

Clube, S.V.M. and Napier, W.M.: 1986, 'Giant comets and the Galaxy: Implications of the terrestrial record', in R. Smoluchowski, J.N. Bahcall and M.S. Matthews (eds.), Univ. Arizona Press, Tucson, 260-295.

Clube, S.V.M. and Asher, D.J.: 1990, 'The evolution of Proto-Encke: Dust bands, close encounters and climatic modulations', in C.I. Lagerkvist, H. Rickman, B.A. Lindblad and M. Lindgren (eds.), Asteroids, Comets, Meteors III, Uppsala, 275-280.

Drummond, J.D.: 1981, 'A test of comet and meteor shower associations', Icarus 45, 545-553.

Galibina, I.V. and Kastel, G.R.: 1982, 'On possible relationship of asteroid (2212) - 1978 SB and $\mathrm{P} /$ Encke comet', Comets and Meteors 33, 45-46 (in Russian).

Jones, J.: 1986, 'The effect of gravitational perturbations on the evolution of the Taurid meteor stream complex', Monthly Not. Roy. Astron. Soc. 221, 257-267.

Kronk, G.W.: 1988, 'Meteor showers: a decriptive catalog', Enslow Publ., Hillside , 296 pp.

Lindblad, B.A.: 1971, 'Two computerized stream searches among meteor orbits', Smithson. Contr. Astrophys. 12, 1-24.

Lindblad, B.A.: 1987, 'The IAU Meteor Data Center in Lund', in Z. Ceplecha and P. Pecina (eds.), Interplanetary Matter, ERAM 10, Publ. Astron. Inst. Czechosl. Acad. Sci.,Praha 2, 201-204.

Lovell, A.C.B.: 1954, 'Meteor astronomy', Oxford Univ. Press, London, 464 pp.

Napier, W.M.: 1983, 'The orbital evolution of short-period comets', in C.I. Lagerkvist and $H$. Rickman (eds.), Asteroids, Comets, Meteors, Univ. Uppsala Press, Uppsala , 391-396.

Olsson-Steel, D.: 1987, 'Asteroid 5025 P-L, comet 1967 II Rudnicki, and the Taurid meteoroid complex', The Observatory 107, 157-160.

Porubčan, V.: 1978, 'Dispersion of orbital elements within the Geminid and Taurid meteor streams', Bull. Astron. Inst. Czechosl. 29, 218-224.

Porubčan, V. and Štohl, J.: 1987a, 'The meteor complex of P/Encke', in Z. Ceplecha and P.Pecina (eds.), Interplanetary Matter, ERAM 10, Publ. Astron. Inst. Czechosl. Acad. Sci.,Praha 2, $167-171$.

Porubčan, V. and Štohl, J.: 1987b, 'On orbits and associations of meteor streams with comets $\mathrm{P} /$ Halley and P/Encke', in B. Battrick and E. Rolfe (eds.), Diversity and Similarity of Comets, ESA SP-278, ESA Publ., ESTEC, Noordwijk , 435-440.

Porubčan, V. and Štohl, J.: 1991, 'Burst of the 1969 Leonids and 1982 Lyrids', in A. Haris and E. Bowell (eds.), Asteroids, Comets, Meteors 1991, Lunar and Planetary Inst. , Houston.

Porubčan, V., Štohl, J. and Van̆a, R.: 1991, 'On associations of Apollo asteroids with meteor streams', in A. Haris and E. Bowell (eds.), Asteroids, Comets, Meteors 1991, Lunar and Planetary Inst. , Houston. 
Sekanina, Z.: 1973, 'Statistical model of meteor streams. III. Stream search among 19303 radio meteors', Icarus 18, 253-284.

Sekanina, Z.: 1976, 'Statistical model of meteor streams. IV. A study of radio streams from the synoptic year', Icarus 27, 265-321.

Steel, D.I., Asher, D.J. and Clube, S.V.M.: 1991, 'The structure and evolution of the Taurid Complex', Monthly Not. Roy. Astron. Soc. , (preprint).

Štohl, J.: 1986, 'The distribution of sporadic meteor radiants and orbits', in C.I. Lagerkvist, B.A. Lindblad, H. Lundstedt and H. Rickman (eds.), Asteroids, Comets, Meteors II, Reprocentralen HSC, Uppsala , 565-574.

Štohl, J. and Porubčan, V.: 1987, 'On applicability of meteor stream membership criteria', in Z. Ceplecha and P. Pecina (eds.), Interplanetary Matter, Publ. Astron. Inst. Czechosl. Acad. Sci., Praha 2, 163-166.

Štohl, J. and Porubčan, V.: 1990, 'Structure of the Taurid meteor complex', in C.I. Lagerkvist, H. Rickman, B.A. Lindblad and M. Lindgren (eds.), Asteroids, Comets, Meteors III, Reprocentralen HSC, Uppsala, 571-574.

Wasson, J.T. and Wetherill, G.W.: 1979, 'Dynamical, chemical and isotope evidence regarding the formation locations of asteroids and meteorites', in T. Gehrels (ed.), Asteroids, Univ. Arizona Press, Tucson, 926-974.

Whipple, F.L.: 1940, 'Photographic meteor studies. III: The Taurid shower', Proc. Amer. Phil. Soc. 83, 711-745.

Whipple, F.L. and Hamid, S.E.: 1952, 'On the origin of the Taurid meteor streams', Helwan Obs. Bull. 41, Harvard Reprint $361,1-30$.

Wright, F.W. and Whipple, F.L.: 1950, 'The photographic Taurid meteors', Tech. Rep. Harvard Coll. Obs., No. 6, (Harvard Reprint Series II-35).

\section{Discussion}

H.U.Keller - You still need high velocities in the order of $3 \mathrm{~km} . \mathrm{s}^{-1}$ even in case of catastrophic disruption of daughter bodies.

J.Štohl - No, on the contrary. Just to avoid high ejection velocities, the assumption of catastrophic disruption of the secondary parent bodies are necessary, or helpful to solve the problem at least.

C.Keay - Do you consider the Tunguska object was a member of the Taurid complex?

J.ŠStohl - Yes. There are good reasons to suppose that the Tunguska object was a member of the Taurid complex in its broadest sense. 